\title{
DBS makes you feel good! - why some of the ethical objections to the use of DBS for neuropsychiatric disorders and enhancement are not convincing
}

\begin{abstract}
Anna Pacholczyk*
Institute for Science, Ethics and Innovation, School of Law, University of Manchester, Manchester, UK

${ }^{*}$ Correspondence: anna.pacholczyk@postgrad.manchester.ac.uk
\end{abstract}

Deep brain stimulation (DBS) offers the potential to relieve some symptoms of movement disorders such as Parkinson's disease and dystonia. Recently, the applications of DBS have been extended to treatment-resistant mental health problems such as depression (Rabins et al., 2009). The successful application of DBS requires stimulation settings to be individually adjusted and the process of finding optimal settings can be lengthy. But what exactly are optimal settings?

Consider a hypothetical case of Philip, a 34-year-old patient with a history of severe depression who undergoes a successful implantation of stimulation electrodes. Depending on the parameters of stimulation, Philip is not clinically depressed but his symptoms drop only to a sub-clinical level (setting 1), he reaches an average level of well-being (setting 2), becomes very cheerful and energetic and takes advantage of it, trying to, as he says, "make up for the lost time" (setting 3), feels even better but a frequent ecstatic state makes him appear to misjudge some situations and he seems to have an incomplete awareness that he may be doing so (setting 4 ), becomes manic to the extent that he is rendered legally incompetent to make treatment decisions (setting 5).

There have been attempts to construct the concepts of disability and disease as a deviation from normal or species-typical functioning (e.g., Sabin and Daniels, 1994). If health is understood as an absence of disease and the goal of a medical intervention is to promote health thus defined, a transition from a diseased state to either a sub-clinical (setting 1) or average state of well-being (setting 2) achieves the goal equally well. However, the "normality view" faces well-known problems and the treatment/enhancement distinction is itself problematic enough not to serve as a good normative guide (Harris, 2007;
Synofzik, 2009). It is unlikely that medical professionals would be satisfied by merely bringing a patient to a sub-clinical state if there is a more effective (after accounting for side-effects) intervention available. And for good reason - at the heart of medical intervention is the concern for the patient's well-being. The effectiveness of DBS for reducing the symptoms as assessed by clinical rating scales is admittedly important, but as Synofzik and Schlaepfer (2008) correctly point out, those improvements have to translate into the patient's improved ability to pursue and achieve their personal goals - goals that are connected to the patient's conception of a good life.

Some consider changes in personality to be among the risks of the DBS procedure (Glannon, 2009). This criticism is problematic even in the case of treatment for movement disorders, as not all side-effects are necessarily unwanted or undesirable, but becomes inapplicable when looking at treatment for neuropsychiatric conditions, since the goal of the intervention is exactly to change some cognitive and affective aspects of personality. Also, the aim of the treatment is not necessarily to rediscover the "real" patient hidden under the symptoms of a disorder. Rather, its aim is to improve patients' quality of life, given their own idea of what that means. Consequently, legally competent patients are not obliged to live up to or agree with the ideas of authenticity or rationality held by their doctors.

Medical professionals may be hesitant to utilize their skills for what they see as enhancement, but this apparent presumption against enhancement seems to be unjustified. Although we may have good reasons to prioritize interventions that improve the lives of those with generally worse health, medical professionals justifiably also use their expertise for medical interventions that have little to do with restoring health. The obvious examples of socially valued enhancements provided by medical professionals are vaccinations (Harris, 2007) and contraception - the goal of the former is to enhance the immune system, while that of the latter is to disrupt the reproductive function, which in turn enhances people's control over their lives. Medical professionals also perform interventions that are of no medical benefit to the subjects of the intervention (for example, blood donation or live organ donation). Thus, there is nothing in principle wrong with doctors using their expertise to provide an enhancing intervention other than treatment. The questions we should be asking instead are about appropriate consent procedures, issues of prioritization, some ethical quandaries arising from the application of DBS and the limits of legitimate power of the physician in the setting characterized by high power inequalities, exacerbated by the fact that a patient cannot simply go to a different health care provider.

Currently, the medical professional may say "we can provide you with the possibility of leading a good life, but not with what you think is necessary for a very good life." However, the presumption should surely be to provide the benefit that the patient seeks, and so the burden of giving reasons against this course of action rests on those who would deny this potential benefit. Legitimate concerns such as those related to limited financial resources (which could be offset if the patient is willing to pay for extra services) or the time constraints of the limited number of DBS specialists have to be weighed against other good moral reasons to perform the enhancing procedure. Some of the variables in this moral calculus may be different in the case of enhancement for non-patients (for example, we have to add a costly and invasive surgical procedure and follow-up), but the principle is the same. Since DBS practitioners are most commonly also researchers and members of 
the scientific community, good reasons in favor of the procedure include, for example, the gains in scientific and medical knowledge about DBS and the brain. If DBS can bring benefit, there have to be really good reasons to deny it.

Although the use of DBS in non-clinical populations is not intrinsically unethical, this scenario seems, at least prima facie, to differ from the clinical uses of DBS in the predicted benefit to the subject and the acceptability of risks. Let us have a closer look. In relation to the use of psychopharmacological agents in non-therapeutic context, Synofzik (2009) proposed that the ethically justified decision of whether to provide and/or recommend a potentially enhancing agent should rest on the assessment of predicted risks and benefits and the respect for autonomy of the subject. His approach has the advantage of accounting not only for the moral weight of the predicted benefits of an intervention (especially when benefits outweigh the risks), but also for the fact that we often (and justifiably so) do what is not in our medical interest or otherwise narrowly construed self-interest in order to live according to our values - and for the importance of respecting choices of those with the capacity to make them, even if we find them surprising or difficult to explain.

Synofzik and Schlaepfer (2008) argued that although there is nothing in principle wrong with DBS for enhancement in non-clinical subjects, DBS is not ready for non-therapeutic application. They point out, for example, that there are no systematic studies of DBS effectiveness with non-clinical populations, making the evidence-based benefit assessment difficult. However, although there have been reports of at least one patient who chooses a setting depending on how she wants to feel (Russo, 2007), there also are no systematic studies of an non-therapeutic use of DBS for clinical populations - but this fact need not be a definitive argument against the permissibility of using DBS in those cases. What seems crucial here is rather the fact that the risks associated with surgery have already been taken, and so the risk/benefit ratio is more favorable in those cases - in other words, "trying it out" seems to be not as risky.

It is worth noting that at present it is highly unlikely that the public will queue to undergo DBS for non-therapeutic purposes - for many the benefits are too uncertain and the invasiveness of the procedure and associated risks and inconveniences too discouraging to even entertain the possibility of undergoing DBS. Those few that want to undergo DBS may do it for reasons that include, but are not limited to, the expectation of benefits. When the expectation of benefits is the main reason, this may be due to misunderstanding about effectiveness and risks of the procedure; this can be however addressed by providing appropriate information and the role of the specialist is of a crucial importance. Potential subjects do not have the obligation to "argue their case" or convince the physician, but they need to display sufficient awareness of the risks and benefits so that the requirements of informed consent are met.

However, there may also be those who are aware of the risks and the speculative nature of benefits but still want to undergo the procedure (e.g., for various reasons to do with pushing the boundaries of science and medicine). What should be done in such cases? Synofzik (2009) suggest that there may be cases where predicted risks outweigh predicted benefits, in which a physician has a good reason to discourage the use of an intervention on medical grounds but may still have a good moral reason to provide it; and to do so on the basis of subjects' widely constructed interest and the respect for their autonomy. So there may be cases, when despite medical risks outweighing the benefits (including cases where risks are possible to estimate with a higher degree of certainty than benefits) it may be ethically permissible to provide DBS solely on the basis of considering the subject's interests, albeit interests that go beyond the simply medical. This first step of the assessment will likely be followed by considering reasons for and against the procedure independent of the first step (funding, the potential to gain knowledge that could be transferred to the clinical setting, progress of science, etc.).

We may understand the sentiment of doctors who want to focus on relieving suffering, but we have to remember that the treatment/enhancement distinction that seems so obvious and apparent in the medical setting is much more difficult to construct as an ethically relevant one. The prospect of non-therapeutic use of DBS, and neuroenhancement in general, raises important questions about the societal implications of neurotechnologies. Among others, it restates old questions about the role and purpose of the medical profession and the role that doctors should play in managing access to medical technologies: those that aim at alleviating suffering and those that more generally promote wellbeing. At present, a limited number of specialists have control over the application of DBS. With this power, however, comes responsibility. Given that the ethics of the neuroenhancing application of DBS is less straightforward than it might have appeared at first sight, this responsibility includes, at the very least, a careful ethical assessment of requests for DBS for non-clinical use.

\section{ACKNOWLEDGMENTS}

I am grateful for the support from the Wellcome Strategic Programme "The Human Body - Its Scope, Limits, and Future." I would also like to thank John Coggon, John Harris, and Tom Lardner for their comments.

\section{REFERENCES}

Glannon, W. (2009). Stimulating brains, altering minds. J. Med. Ethics 35, 289-292.

Harris, J. (2007). Enhancing Evolution. Princeton: Princeton University Press.

Rabins, P., Appleby, B., Brandt, J., DeLong, M. R., Dunn, L. B., Gabriëls, L., Greenberg, B. D., Haber, S. N., Holtzheimer, P.E., Mari, Z., Mayberg, H. S., McCann, E., Mink, S. P., Rasmussen, S., Schlaepfer, T. E., Vawter, D. E., Vitek, J. L., Walkup, J., and Mathews, D. J. H. (2009). Scientific and ethical issues related to deep brain stimulation for disorders of mood, behavior and thought. Arch. Gen. Psychiatry 66, 931-937.

Russo, F. (2007). How to change a personality. Time 169, 101.

Sabin, J.E., and Daniels, N. (1994). Determining "medical necessity" in mental health practice. Hastings Cent. Rep. 24, 5-13.

Synofzik, M. (2009). Ethically justified, clinically applicable criteria for physician decision-making in psychopharmacological enhancement. Neuroethics 2, 89-102. Synofzik, M., and Schlaepfer, T. E. (2008). Stimulating personality: ethical criteria for deep brain stimulation in psychiatric patients and for enhancement purposes. Biotechnol. J. 3, 1511-1520.

Received: 08 January 2011; accepted: 18 April 2011; published online: 06 May 2011.

Citation: Pacholczyk A (2011) DBS makes you feel good! - why some of the ethical objections to the use of DBS for neuropsychiatric disorders and enhancement are not convincing. Front. Integr. Neurosci. 5:14. doi: 10.3389/ fnint.2011.00014

Copyright (ㄷ) 2011 Pacholczyk. This is an open-access article subject to a non-exclusive license between the authors and Frontiers Media SA, which permits use, distribution and reproduction in other forums, provided the original authors and source are credited and other Frontiers conditions are complied with. 